\title{
Dynamic Analyses of Six Degree Freedom Platform Based on Improvement of PID Neural Networks
}

\author{
Sujian Sheng ${ }^{1}$, Bo Yang ${ }^{1}$, Pinle Qin ${ }^{2}$, Xiaoqing Chen ${ }^{2}$ \\ 1 CNOOC Energy Technology \& Services- Oil Production Services Co. \\ 2 Department of Computer and Control Engineering, North University of China, Taiyuan 030051 \\ China \\ Pinle.qin@dlut.edu.cn
}

\begin{abstract}
In this paper, traditional PID neural network (PIDNN) control theory was introduced and the characteristics and disadvantages of the algorithm was been analysis. An improvement PIDNN method based on multi-innovation theory was proposed. Then the steward tracking control system was simulated, some planning trace was given out and the math model of the control system was established. The result showed that the improvement algorithm is effect.
\end{abstract}

\section{Introduction}

The actual systems of factories are usually very complex, which owns features of nonlinear and time-varying. We have to build system model if we want to control them. But the accurate ones are impossible. Then we need input-output data of the system to identify and get the approximate model.

Based on neural network of nonlinear identification method is a common method which is simple as well. It does not need so many prior experiences, we are able to modify weight and finally identify systems only through learning and practicing. This provides an effective way to identify nonlinear system.

PID neural network (PIDNN) [1] which is proposed by SHU et al. (2000) is a new kind of networks and its hidden layer neurons simply work as PID controller terms through their activation functions thus it simultaneously utilizes advantages of both PID controller and neural structure. Due to the introduction of proportional (P), integral (I), differential (D), PID neural network has dynamic feature. Therefore it is suitable for the identification of dynamic system.

However, PID neural network use the batch mode of BP algorithm to modify weight, which is incommodious to identify systems in real time. Paper [3] suggests that the online mode of BP algorithm is more scientific and efficient than batch mode about the aspect of realtime identification and control in the system.

The online mode of BP algorithm uses the current input-output data only when it adjusts network weight, but not the past ones. The multi-innovation identification algorithms which is presented by Ding use not only the current data but also the past data at each iteration, parameter estimation accuracy can be improved. Enlightened by paper [4], this article introduces the current input-output data into PIDNN, presents the multi-innovation learning algorithm based on PID neural network and shows convergence proof. Simulation and experimental results demonstrate that the multi-innovation learning algorithm based on PID neural network has better performance in system identification.

\section{PID neural network model and Multi-innovation identification algorithm}

\section{A. PID neural network model}

Consider a time-invariant stochastic system described by a linear regression model:

$$
y(t)=\varphi^{T}(t) \theta+v(t)
$$

Where $\theta \in R^{n}$ is the parameter vector of system, $y(t) \in R^{1}$ is the system output, $u(t) \in R^{1}$ is 
the system input, $v(t) \in R^{1}$ is a stochastic noise with zero mean, $\varphi(t) \in R^{n}$ is the information vector consisting of the system observation (input-output) data, the superscript $T$ denotes the transpose.

As it is shown in figure 1, PID neural network has a simple feed forward neural network which consists of 2-3-1 structure, so it has three layers. Let $W=\left(w_{i j}\right)_{2 \times 3}$ be the weight matrix between the input layer and the hidden layer and $V=\left(v_{1}, v_{2}, v_{3}\right)$ be the weight matrix between the hidden layer and the output layer. $u_{i}, u_{j}^{\prime}$ and $u^{\prime \prime}$ are the inputs of the input layer, the hidden layer and the output layer. $x_{i}, x_{j}^{\prime}$ and $x^{\prime \prime}$ are the outputs of them. The actual output of the PID neural network is $d$.

We can use it to approximate the process described by expressing (1).

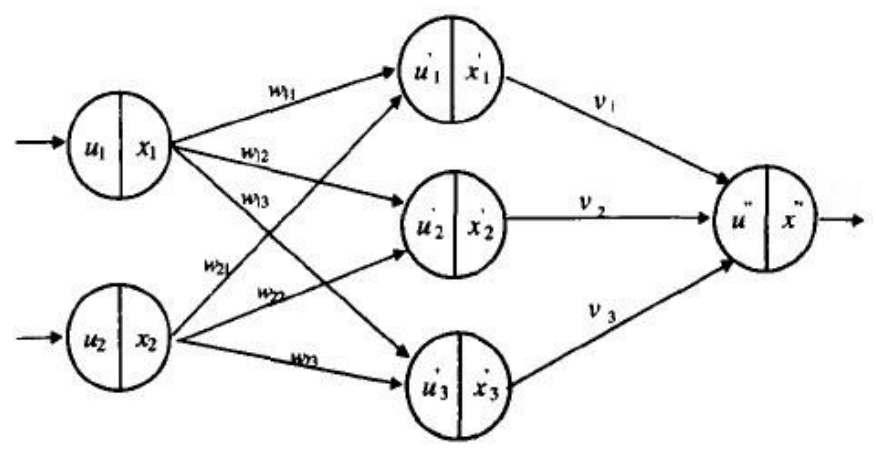

Figure 1. Structure of PIDNN

\section{B. Multi-innovation identification algorithm}

The following single innovation identification algorithm can be used to estimate the parameter $\theta$ of system (1).

$$
\begin{gathered}
\hat{\theta}(t)=\hat{\theta}(t-1)+\frac{\varphi(t)}{r(t)} e(t) \\
e(t)=y(t)-\varphi^{T}(t) \hat{\theta}(t-1) \\
r(t-1)=r(t-1)+\|\varphi(t)\|^{2} \quad, \quad r(0)=1
\end{gathered}
$$

here, $e(t)$ is a single innovation.

In multi-innovation identification algorithm, the single innovation $e(t) \in R^{1}$ is extended to multi-innovation vector $E(p, t-1)=[e(t-1), e(t-1), \cdots, e(t-p)]^{T} \in R^{p}$, the information vector $\varphi(t) \in R^{n}$ is also extended to $\Phi(t) \in R^{n \times p}$ for scalar system. From here , the algorithm can be expressed as

$$
\hat{\theta}(t)=\hat{\theta}(t-1)+\frac{\Phi(p, t)}{r(t)} E(p, t)
$$

where $p \geq 1$ represents the innovation length. As $p=1$, the multi-innovation identification algorithm reduces to the single innovation identification algorithm.

\section{Mutil-innovation learning algorithm based on PID neural network}

Considering $p$ groups of input-output data from time $t-p+1$ to time $t$, the output vectors of the $i$ th input node is

$$
X_{i}(p, t)=\left[x_{i}(t), x_{i}(t-1), \cdots, x_{i}(t-p+1)\right], \quad i=1,2
$$

The output vector of the output node is

$$
D(p, t)=[d(t), d(t-1), \cdots d(t-p+1)]^{T}
$$


The expected vector of PID neural network is

$$
Y(p, t)=[y(t), y(t-1), \cdots, y(t-p+1)]^{T}
$$

Define a cost function:

$$
J(p, t)=\frac{1}{2}\|Y(p, t)-D(p, t)\|^{2}
$$

Thus, we proceed to refine the weights by the training iteration as follows:

$$
\begin{gathered}
v_{j}(t+1)=v_{j}(t)-\eta \frac{\partial J(p, t)}{\partial v_{j}} \\
w_{i j}(t+1)=w_{i j}(t)-\eta \frac{\partial J(p, t)}{\partial w_{i j}}
\end{gathered}
$$

where $\eta>0$ is the learning rate,

$$
\begin{aligned}
\frac{\partial J(p, t)}{\partial v_{j}} & =\frac{\partial X^{\prime \prime}(p, t)}{\partial v_{j}} \frac{\partial J(p, t)}{\partial X^{\prime \prime}(p, t)} \\
& =\left[x_{j}^{\prime}(t), \cdots, x_{j}^{\prime}(t-p+1)\right]\left[\frac{\partial J(p, t)}{\partial x^{\prime \prime}(t)}, \cdots, \frac{\partial J(p, t)}{\partial x^{\prime \prime}(t-p+1)}\right]^{T} \\
& =X_{j}^{\prime}(p, t)\left[\frac{\partial J(p, t)}{\partial d(t)}, \cdots, \frac{\partial J(p, t)}{\partial d(t-p+1)}\right]^{T} \\
& =X_{j}^{\prime}(p, t)[-e(t), \cdots,-e(t-p+1)]^{T} \\
& =-X_{j}^{\prime}(p, t) E(p, t)
\end{aligned}
$$

and

$$
\begin{aligned}
\frac{\partial J(p, t)}{\partial w_{i j}} & =\frac{\partial U_{j}^{\prime}(p, t)}{\partial w_{i j}} \frac{\partial J(p, t)}{\partial U_{j}^{\prime}(p, t)} \\
& =\left[x_{i}(t), \cdots, x_{i}(t-p+1)\right]\left[\frac{\partial J(p, t)}{\partial u_{j}^{\prime}(t)}, \cdots, \frac{\partial J(p, t)}{\partial u_{j}^{\prime}(t-p+1)}\right]^{T} \\
& =X_{i}(p, t)\left[\frac{\partial J(p, t)}{\partial d(t)} \frac{\partial d(t)}{\partial x_{j}^{\prime}(t)} \frac{\partial x_{j}^{\prime}(t)}{\partial u_{j}^{\prime}(t)}, \cdots, \frac{\partial J(p, t)}{\partial u_{j}^{\prime}(t-p+1)}\right]^{T} \\
& =X_{i}(p, t)\left[-e(t) v_{j}(t) \operatorname{sign} \frac{x_{j}^{\prime}(t)-x_{j}^{\prime}(t-1)}{u_{j}^{\prime}(t)-u_{j}^{\prime}(t-1)}, \cdots, \frac{\partial J(p, t)}{\partial u_{j}^{\prime}(t-p+1)}\right]^{T} \\
& =X_{i}(p, t)\left[-e_{1}(t), \cdots,-e_{1}(t-p+1)\right] \\
& =-X_{i}(p, t) E_{1}(p, t)
\end{aligned}
$$

So we have

$$
v_{j}(t+1)=v_{j}(t)+\eta X_{j}^{\prime}(p, t) E(p, t)
$$

and

$$
w_{i j}(t+1)=w_{i j}(t)+\eta X_{i}(p, t) E_{1}(p, t)
$$

\section{Convergence analysis of multi-innovation learning algorithm}

For the identification of nonlinear dynamical systems, PID neural network can be treated as a dynamical system described by (1). Its adjustable parameters $\theta=\left(w_{i j}, v_{j}\right)$ which is made of $W=\left(w_{i j}\right)_{2 \times 3}$ and $V=\left(v_{1}, v_{2}, v_{3}\right)$ can be written as (5). 
Reference [5], convergence analysis of the proposed algorithm be given as fellows.

Theorem 1: For the system in (1), assume that a stochastic white noise with zero mean $v(t)$ is uncorrelated with $u(t)$

(A1) $E\left[v^{2}(t)\right] \leq \sigma_{v}^{2}<\infty ;$

if there exist constants $0<\alpha \leq \beta<\infty$ and the innovation length $p \geq n$ such that the following persistent excitation condition holds,

(A2) $\alpha I \leq \frac{1}{p} \sum_{i=1}^{p} \varphi(t-i+1) \varphi^{T}(t-i+1) \leq \beta I ;$

Then the parameter estimation $\hat{\theta}(t)$ given by Eq.(5) is uniform bounded.

Proof:

Define the estimation error $\tilde{\theta}(t)=\theta(t)-\theta$, assume $\theta$ is real value. Subtracting $\theta$ from the two sides of Eq.(5), we have

$$
\begin{aligned}
& \theta(t)-\theta=\theta(t-1)-\theta+\frac{\Phi(p, t)}{r(t)}\left[\Phi^{T}(p, t) \theta+V(p, t)-\Phi^{T}(p, t) \theta(t-1)\right] \\
& =\theta(t-1)-\theta+\frac{\Phi(p, t)}{r(t)}\left[-\Phi^{T}(p, t)(\theta(t-1)-\theta)+V(p, t)\right]
\end{aligned}
$$

Substituting $\tilde{\theta}(t)$

$$
\begin{aligned}
\tilde{\theta}(t) & =\tilde{\theta}(t-1)+\frac{\Phi(p, t)}{r(t)}\left[-\Phi^{T}(p, t) \tilde{\theta}(t-1)+V(p, t)\right] \\
& =\left[I-\frac{\Phi(p, t) \Phi^{T}(p, t)}{r(t)}\right] \tilde{\theta}(t-1)+\frac{\Phi(p, t) V(p, t)}{r(t)}
\end{aligned}
$$

By using A2, taking the trace gives

$$
\begin{aligned}
& p \alpha \leq \frac{1}{p} \sum_{i=1}^{p}\|\varphi(t-i+1)\|^{2} \leq p \beta \\
& n p \alpha \leq \sum_{i=1}^{p}\|\varphi(t-i+1)\|^{2} \leq n p \beta
\end{aligned}
$$

Hence, we have

$$
n p \alpha \leq\|\varphi(t)\|^{2} \leq n p \beta
$$

According to the definition of $r(t)$ in (4), we have

$$
\begin{aligned}
r(t) & =\sum_{j=1}^{t}\|\varphi(j)\|^{2}+r(0) \\
& \leq \sum_{j=0}^{\lfloor(t-1) / p\rfloor} \sum_{i=1}^{p}\|\varphi(j p+i)\|^{2}+r(0) \\
& \leq \sum_{j=0}^{\lfloor(t-1) / p\rfloor} n p \beta+1 \\
& \leq(\lfloor(t-1) / p\rfloor+1) n p \beta+1 \\
& \leq(t+p-1) n \beta+1
\end{aligned}
$$




$$
\begin{aligned}
r(t) & =\sum_{j=1}^{t}\|\varphi(j)\|^{2}+r(0) \\
& \geq \sum_{j=0}^{\lfloor t / p\rfloor-1} \sum_{i=1}^{p}\|\varphi(j p+i)\|^{2}+r(0) \\
& \geq \sum_{j=0}^{\lfloor t / p\rfloor-1} n p \alpha+r(0) \\
& \geq(\lfloor t / p\rfloor-1+1) n p \alpha+1 \\
& \geq \operatorname{tn} \alpha+1
\end{aligned}
$$

Then, the following inequality has been obtained

$$
\begin{gathered}
\operatorname{tn} \alpha+1 \leq r(t) \leq(t+p-1) n \beta+1 \\
I-\frac{\Phi(p, t) \Phi^{T}(p, t)}{r(t)} \leq\left[1-\frac{p \alpha}{(t+p-1) n \beta+1}\right] I
\end{gathered}
$$

Here, using assume A1, we have

$$
\begin{aligned}
E(\|\Phi(p, t) V(p, t)\|) & \leq \sqrt{E\left(\|\Phi(p, t) V(p, t)\|^{2}\right)} \\
& \leq \sqrt{p \beta E\left(\|V(p, t)\|^{2}\right)} \\
& \leq \sqrt{p \beta p \sigma_{v}^{2}} \\
& \leq p \sigma_{v} \sqrt{\beta}
\end{aligned}
$$

Taking the expectation and norm of (38) and using (39) give

$$
\begin{aligned}
& \|\tilde{\theta}(t)\|=\left\|\left[-\frac{\Phi(p, t) \Phi^{T}(p, t)}{r(t)}\right] \tilde{\theta}(t-1)+\frac{\Phi(p, t) V(p, t)}{r(t)}\right\| \\
& \leq\left\|\left[I-\frac{\Phi(p, t) \Phi^{T}(p, t)}{r(t)}\right] \tilde{\theta}(t-1)\right\|+\left\|\frac{\Phi(p, t) V(p, t)}{r(t)}\right\| \\
& \leq\left\|\left[1-\frac{p \alpha}{(t+p-1) n \beta+1}\right]\right\|\|\tilde{\theta}(t-1)\|+\frac{\|\Phi(p, t) V(p, t)\|}{r(t)} \\
& \leq\left\|\left[1-\frac{p \alpha}{(t+p-1) n \beta+1}\right]\right\|\|\tilde{\theta}(t-1)\|+\frac{\|\Phi(p, t) V(p, t)\|}{r(t)} \\
& \leq\left\|\left[1-\frac{p \alpha}{(t+p-1) n \beta+1}\right]\right\|\|\tilde{\theta}(t-1)\|+\frac{\|\Phi(p, t) V(p, t)\|}{\operatorname{tn} \alpha+1} \\
& E(\|\tilde{\theta}(t)\|) \leq\left[1-\frac{p \alpha}{(t+p-1) n \beta+1}\right] \| E(\|\tilde{\theta}(t-1)\|)+\frac{1}{t n \alpha+1} E(\|\Phi(p, t) V(p, t)\|) \\
& \leq\left\|\left[1-\frac{p \alpha}{(t+p-1) n \beta+1}\right]\right\| E(\|\tilde{\theta}(t-1)\|)+\frac{p \sigma_{v} \sqrt{\beta}}{t n \alpha+1}
\end{aligned}
$$

If we select suitable parameter $\alpha$ and $\beta$, it follows that

where $d$ is a constant. Thus,

$$
0<1-\frac{p \alpha}{(t+p-1) n \beta+1} \leq d<1
$$

$$
E(\|\tilde{\theta}(t)\|) \leq d E(\|\tilde{\theta}(t-1)\|)+M
$$

where $M$ is a constant. Repeating using the formula 


$$
\begin{aligned}
E(\|\tilde{\theta}(t)\|) & \leq d E(\|\tilde{\theta}(t-1)\|)+M \\
& \leq d^{t-1} E(\|\tilde{\theta}(1)\|)+\frac{1-d^{t-1}}{1-d} M
\end{aligned}
$$

This completes the proof of Theorem 1.

\section{The Stewart System Analysis}

Ships and offshore platforms have been widely used in the marine transportation and exploitation of ocean resources. Due to the load of wind, wave, current of sea , The platform will occur oscillating movement, Not only have effects on personnel health and equipment, but do harm to the offshore production. Stewart platform has six degrees of freedom of movement parallel mechanism with advantages about stiffness, bearing capacity, high accuracy. this thesis combine the characteristics of ocean wave motion and parallel institutions, Design and analysis a Ship borne compensated stable platform based on 6-SPU Stewart mechanism. The construction of the system show as the Fig.1.
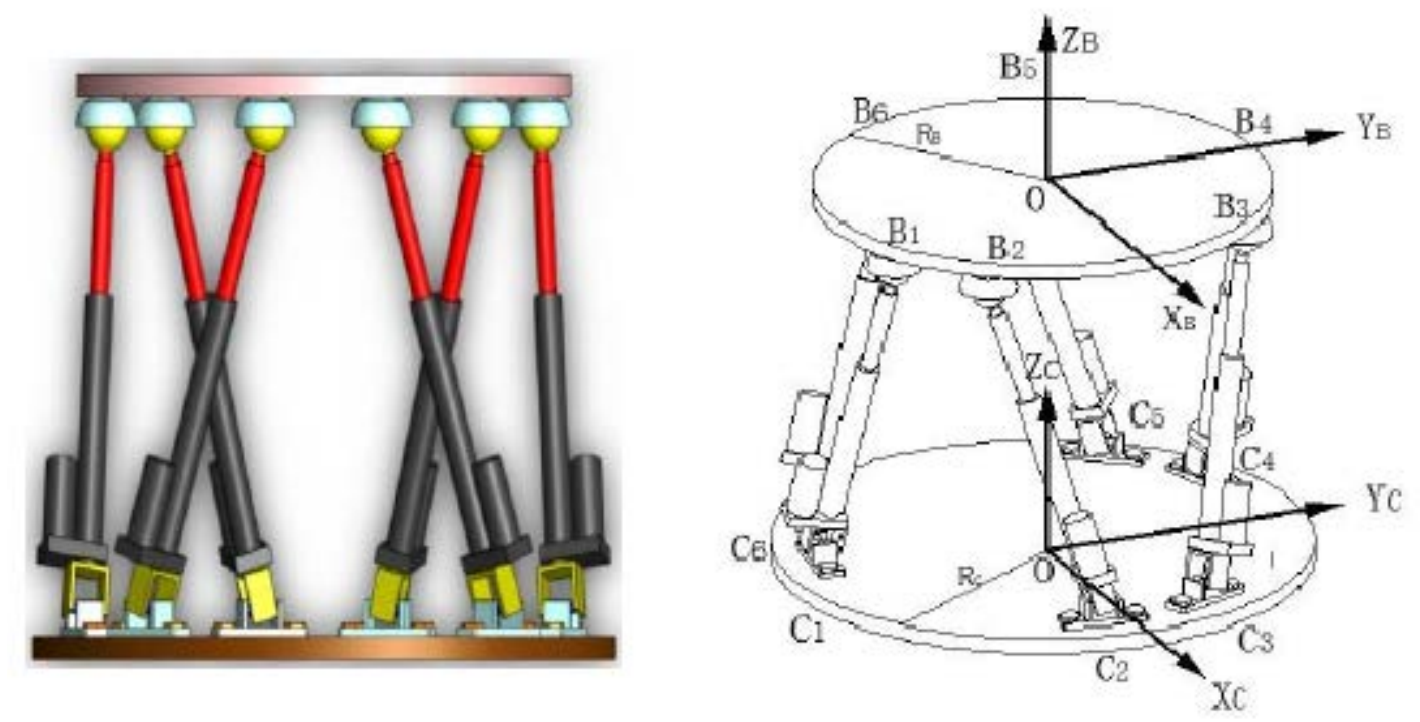

Figure 2. the construction of stewart motion system

According to the space coordinate transformation, the vector $\mathrm{R}^{\prime}$ of the moving coordinate system can be transformed the vector $\mathrm{R}$ of static coordinate system.

$$
\begin{aligned}
& R=T R^{\prime}+P \\
& T=\left[\begin{array}{lll}
d_{11} & d_{12} & d_{13} \\
d_{21} & d_{22} & d_{23} \\
d_{31} & d_{32} & d_{33}
\end{array}\right] \\
& P=\{X c, Y c, Z c\}^{T}
\end{aligned}
$$

\section{Examples}

To illustrate the effectiveness of the proposed algorithm, this paper compares its accuracy with the traditionary BP algorithm in the control of stewart motion platform. The test signal used for the example is

$$
u(t)=0.2 \sin \frac{2 \pi t}{25}+0.3 \sin \frac{\pi t}{75}
$$

The initial weight matrix in output layer $W(0)=[1,0.1,1 ;-1,-0.1,-1]$, the initial weight matrix 
in hidden layer $V(0)=(0.1,0.1,0.1)$, the innovation length $p=2$, the learning rate $\eta=0.175$. After 20 learning steps, the system responses are shown in Fig 3 and Fig 4.

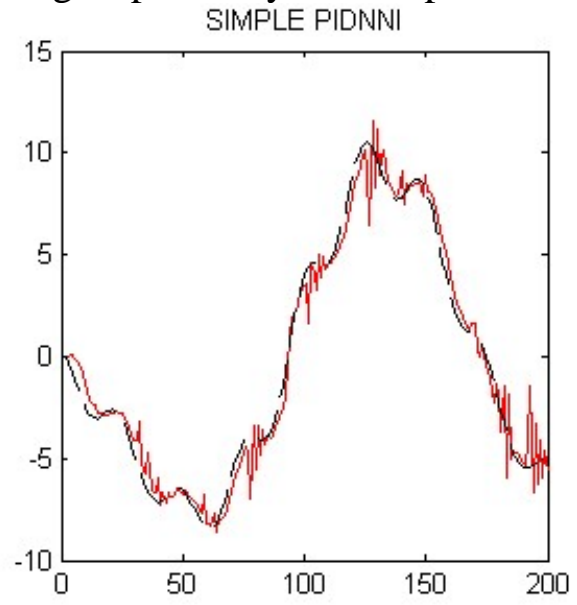

Fig 3.Identification effect of the unmodified algorithm

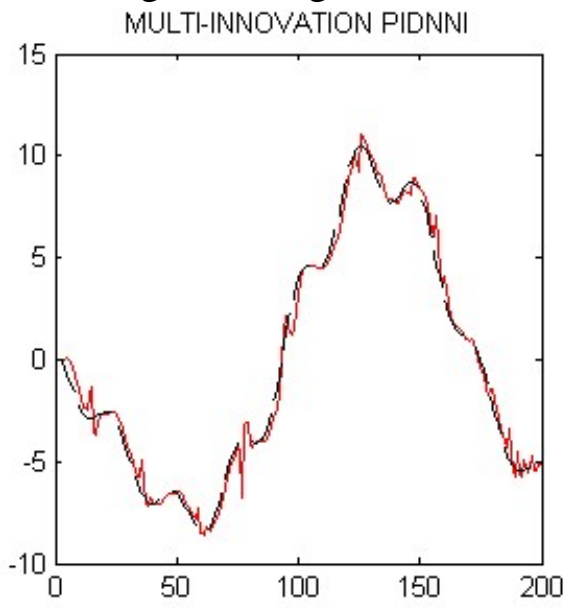

Fig 4.Identification effect of the proposed algorithm

Example 2. Considering the of the stewart model systems described by the following function[2].

where $f[y(t)]=\frac{5 y(t)}{2.5+y^{2}(t)}, \quad g[u(t)]=u^{3}(t)$

The test signal used for the example is

$$
u(t)=0.6 \sin \frac{2 \pi t}{50}+0.4 \sin \frac{2 \pi t}{75}
$$

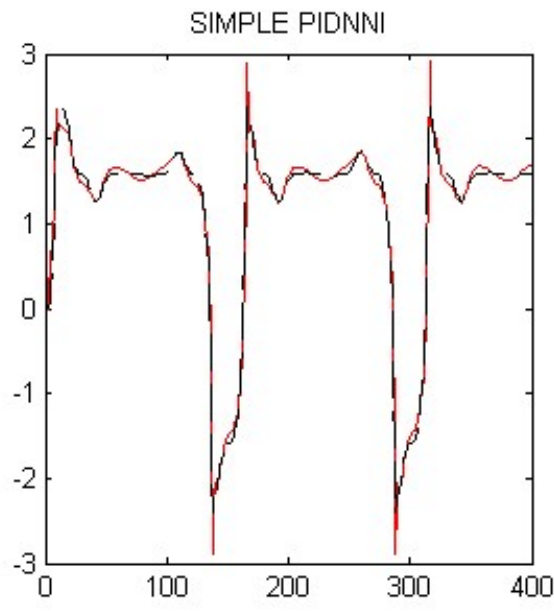

Fig 5.Identification effect of the unmodified algorithm

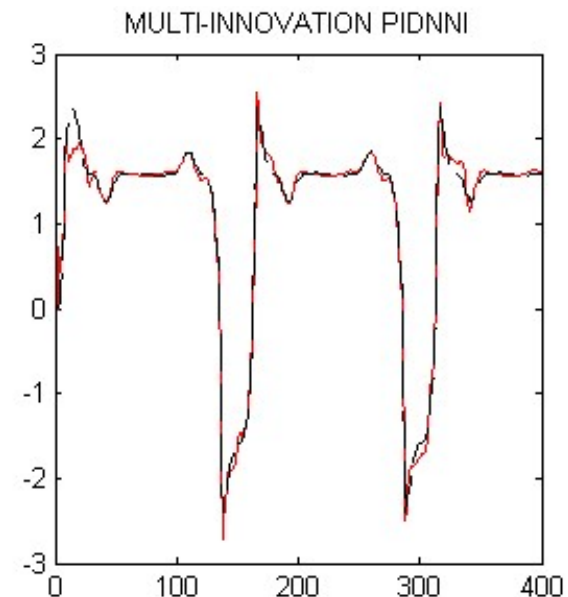

Fig 6.Identification effect of the proposed algorithm

Here, the initial weight matrices of PID neural network be the same as the above example, the innovation length $p=3$, the learning rate $\eta=0.015$. After 20 learning steps, the system responses are shown in Fig 5 and Fig 6.

\section{Conclusion}

This paper proposed a multi-innovation learning algorithm based on PID neural network and analyses the convergence of the algorithm. From the application simulation of stewart platform motion control, it is shown that the proposed algorithm has fast convergence rate and good tracking ability. 


\section{References}

[1] Huailin,S.and Pi,Y.,PID neural networks for time-delay systems,Computers and Chemical Engineering, 2000,24:859-862,.

[2] Bhaskar Dasgupta, Prasun Choudhury. A General Strategy Based on the Newton-Euler Approach for the Dynamic; Formulation of Parallel Manipulators[J]. Mechanism and Machine Theory 1999, 34 (6): 801-824

[3] Multi-innovation stochastic gradient identification algorithm based on feedforward neural networks,Journal of Harbin University of Commerce (Natural Sciences Edition),2006, 22(2):123-129.

[4]Feng Ding,Tongwen Chen.Performance analysis of multi-innovation gradient type identification methods,Automatica. 2009, 4:1-14.

[5] T. A. Dwarakanath, Bhaskar Dasgupta, T. S. Mruthyunjaya. Design and Development of a Stewart Platform based Force-Torque Sensor,2001,(11):793-809

[6]Shu Huai lin. Iden Tification of Nonlinear Systems Based on PID Neural Networks. Act A Automatic Sinic A. 2002,28(3):474-476. 